\title{
THE INFLUENCE OF IODINE ON THE EXCRETION OF CREATINE IN EXOPHTHALMIC GOITER ${ }^{1}$
}

\author{
WALTER W. PALMER, DONALD A. CARSON, AND LAWRENCE W. SLOAN \\ (From the Department of Medicine, College of Physicians and Surgeons, Columbia \\ University, and the Presbyterian Hospital, New York)
}

(Received for publication August 29, 1928)

Normal human adults taking a diet without meat, fish, peas or beans excrete no creatine in the urine. The exceptions to this thus far observed are the appearance of small amounts of creatine in women during the menstrual period (1) and the creatinuria in women reported by Denis and Minot (2) following the ingestion of a high protein diet. Creatine is found in the urine of individuals suffering from wasting diseases, starvation, during carbohydrate deprivation, diabetes mellitus, poisoning by phlorhizin, hydrazine, adrenalin and sodium selenite, exophthalmic goiter, fevers and the muscular atrophies and dystrophies (3). The presence of creatine in the urine in pathological conditions is considered evidence of abnormal endogenous nitrogen metabolism of muscles. Shaffer (4) reports 0.18 gram of creatine nitrogen in the twenty-four-hour urine of patients ill with exophthalmic goiter. Of the several pathological conditions studied by Shaffer only in the post partum period did he encounter values in excess of this. Associated with the abnormal creatine excretion in exophthalmic goiter there was a reduced excretion of creatinine. Normally the daily excretion of creatinine nitrogen per kilogram of body weight amounts to 7 to $11 \mathrm{mgm}$., whereas in the cases of goiter only 3 to $6 \mathrm{mgm}$. are excreted.

Since the creatine-creatinine metabolism is apparently of great significance in normal muscle function the known disturbance of this phase of metabolism in exophthalmic goiter on the one hand and the effect of iodine administration in reducing basal metabolism and im-

${ }^{1}$ A brief abstract of this work was presented before the Society for Experimental Biology and Medicine. Proc. Soc. Exp. Biol. and Med., 1927, xxv, 229. 
provement clinically on the other, it seemed desirable to study further the creatine-creatinine metabolism in this disease as influenced by the administration of iodine. Furthermore, the intimate association of creatine and creatinine with muscle function and the important and striking symptom of muscular weakness in this disease add interest to the observation we desire to report.

\section{EXPERIMENTAL}

Cases of exophthalmic goiter were studied in a specially organized metabolism ward in order to insure proper dietary control and accurate collection of urinary specimens. The diets employed were creatinefree with a caloric value equivalent to 100 per cent above the actually determined basal requirement. The protein was fixed between 1 and $1 \frac{1}{2}$ grams per kilogram, the carbohydrate and fat distributed according to the individual preferences of the subject. Invariably the carbohydrates were more freely taken than the fats. Such a diet in our experience has established nitrogen equilibrium or a positive nitrogen balance within three or four days. After a period of three days to a week iodine was administered as Lugol's solution in 1 to $3 \mathrm{cc}$. amounts daily, in practically all instances as part of the preparation for partial thyroidectomy. Creatine and creatinine were determined by the usual Folin method. All specimens of urine were examined for ketone bodies before the determinations were made. At the outset during the winter 1925-1926 uric acid and nitrogen balances were determined. Later these observations were discontinued for reasons given later.

Eight of the cases reported in this paper were studied by D. A. C. in the Lane and Stanford University Hospital in San Francisco during the winter of 1927 and 1928. Some of these patients received iodine in the form of sodium iodide either alone or in association with Lugol's solution.

\section{RESULTS}

For convenience in discussion the 43 cases studied are divided into two groups and arranged in order of the elevation of the basal metabolic rate in tables 1 and 2. The first group, table 1, includes the cases quite generally recognized as true exophthalmic goiter, and the second group in table 2 the subjects, as a rule older, without exophthalmos 
and with an asymmetrically enlarged nodular thyroid gland frequently designated "toxic adenomas." With one exception we have included only those patients who had a partial thyroidectomy. The reports of the surgical pathologist correspond with the above grouping. Six cases were not included in this report because operation was refused or they were otherwise incomplete. However, nothing was observed in the partial studies of these subjects that is inconsistent with the results obtained in the completed cases.

We do not consider it necessary to report all 43 experiments in detail since the space required for this would be unreasonable and we believe it is possible to present the essential features in condensed form without invalidating the conclusions reached.

In group I in every instance with one exception, where appreciable amounts of creatine were excreted in the urine, within from 3 to 7 days after beginning the administration of iodine there was a decided and quite spectacular diminution in the creatinuria. After receiving $10 \mathrm{mgm}$. of iodine in the form of $\mathrm{NaI}$ for six days case 22 shows no reduction in the amount of creatine excreted. It is unfortunate she was operated on without a longer period of iodine intake. Cases 17, 24,28 and 29 excreted small amounts of creatine throughout the preoperative course and a trace of creatine persisted in several of the subjects until the day of operation.

No very definite relation between the increase in basal metabolism and creatinuria exists, although in those cases with a basal rate below +30 per cent the daily excretion of creatine is relatively small. The effect of the iodine administration in reducing the basal rate was considered a possible explanation of the reduction in the creatinuria. While this is observed in most of the subjects, cases 4 and 27 are striking exceptions. In view of the presence of creatine in the urine of children, age was considered as a factor in the prevalence of creatinuria in hyperthyroidism. When the ages are plotted against the preiodine creatine amounts there is little correlation found. It is true that the individuals 38 and over excreted relatively less creatine than those younger. No relationship seems to exist between the age, severity of the disease and the rapidity with which the response to iodine occurs. No difference in behavior of males and females can be 


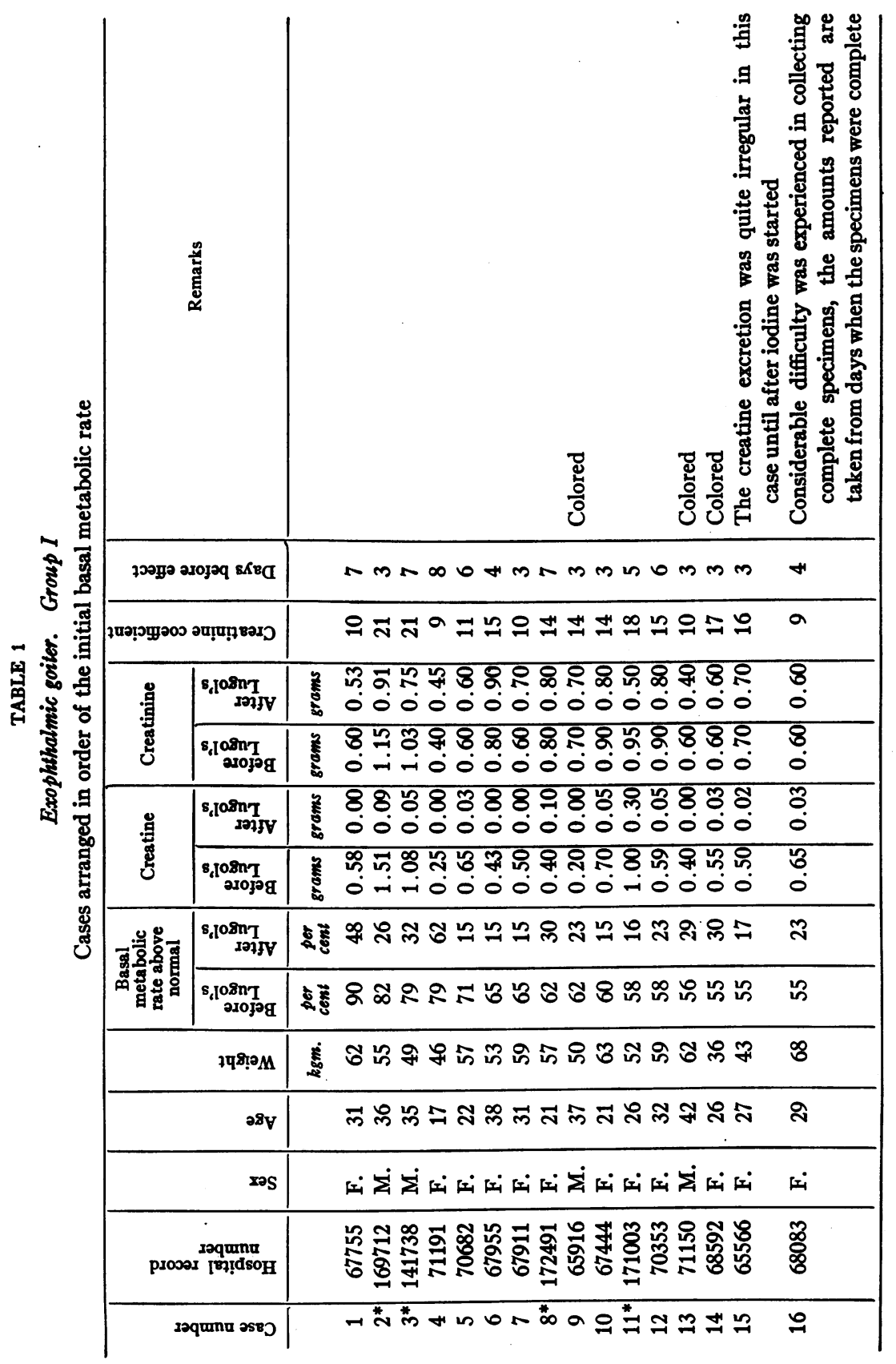




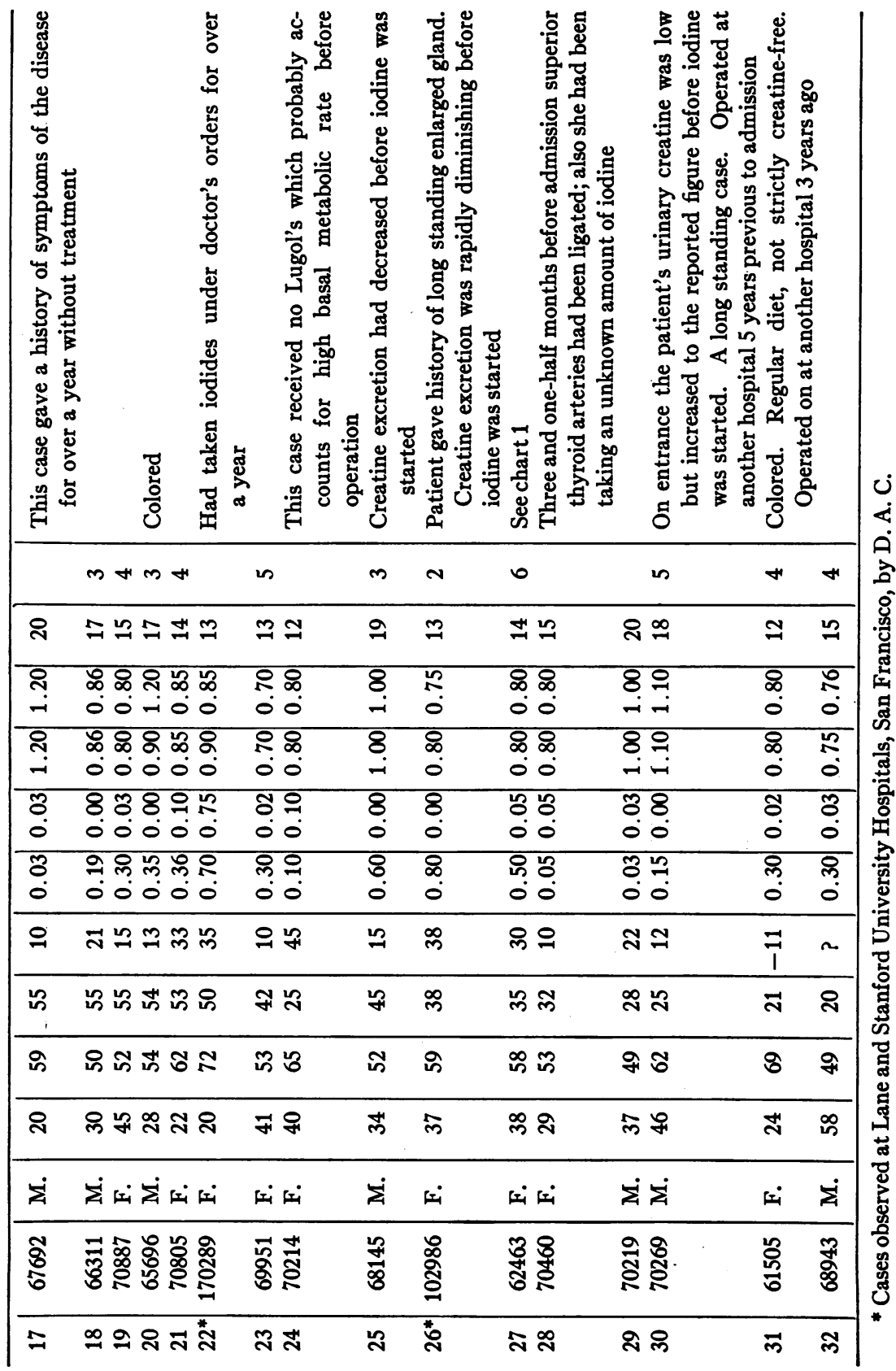




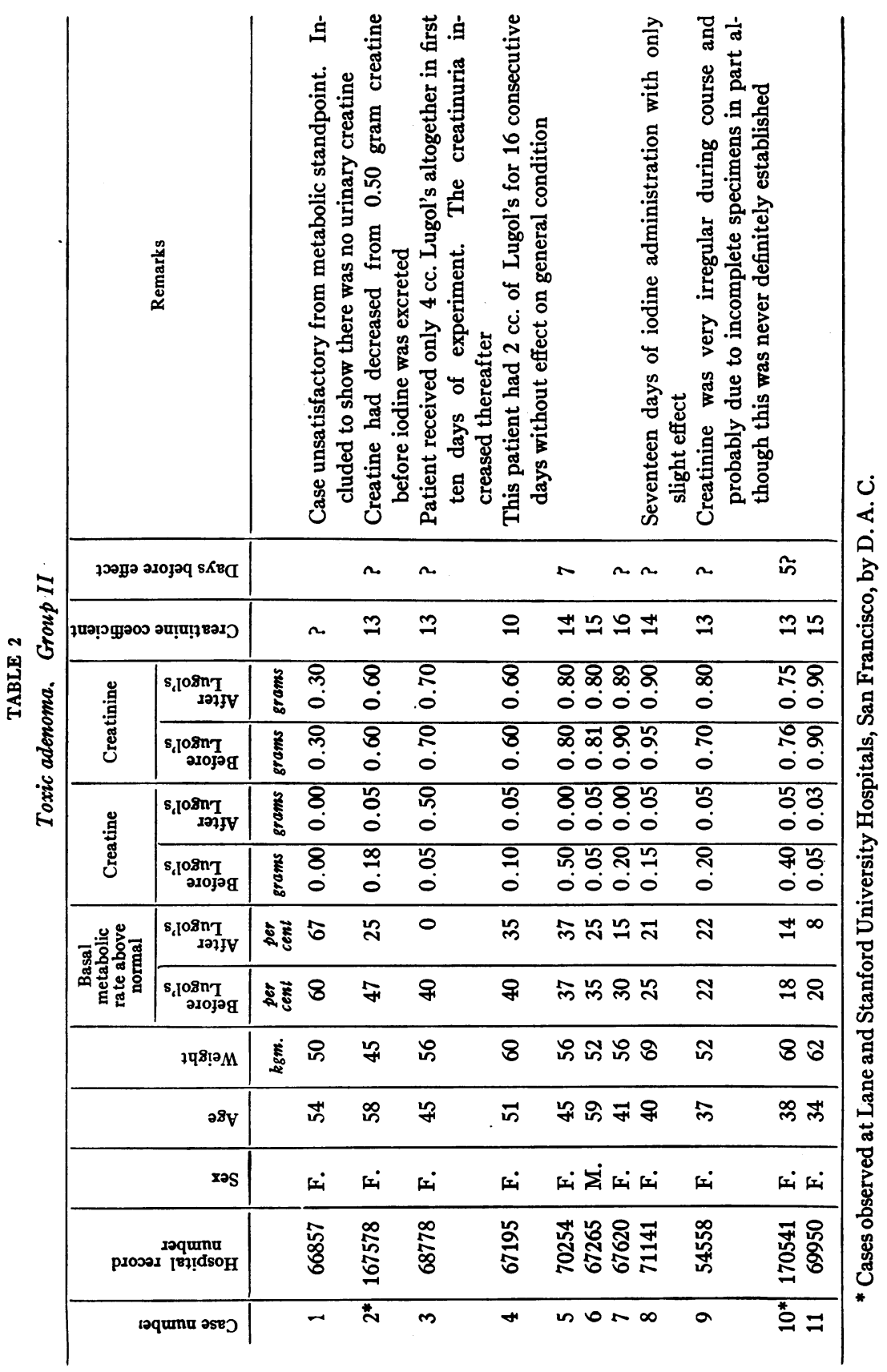


observed despite the fact that normal females can be made to excrete creatine on a creatine-free high protein diet and males cannot (2).

The creatinine excretion is lower than normal. Our creatinine coefficients fall within the identical limits of those observed by Shaffer (4). Expressed as milligrams creatinine nitrogen per kilogram they vary from 3 to 6 . In most instances the administration of iodine seems to effect the creatinine very little. A few cases reveal a slight reduction, in others an increase. We were able to discover no increase in creatinine excretion commensurate with the creatine reduction after the administration of iodine. During the period before iodine was given we frequently noted variations in creatinine excretion not encountered in normal individuals. We feared at first this might be due to incomplete specimens but careful investigation showed this not to be the case. Plotting the age against the creatinine coefficient failed to reveal any positive relation.

Group II contains only eleven cases but there is one fairly outstanding difference between this group and the first group in its behavior following iodine administration. In all the cases the amounts of creatine excreted are small, amounting to mere traces in many. The cases excreting the larger amounts show a fäirly definite diminution of the creatinuria following iodine intake. Otherwise the "toxic adenoma" group reveal little that does not coincide with the results observed in the exophthalmic group. As a group the cases are older only one aged 34, the others 37 or over, while in Group I, 24 were 37 or under, the youngest aged 17. There was only one male in the group.

In chart 1 is presented graphically the details of case 27 of Group I which was observed first during eleven days of iodine administration which seemed to have little if any effect on the clinical picture and no influence on the basal rate. The patient did not wish an operation; iodine was discontinued for six weeks, resumed later in preparation for operation. The behavior of creatinuria in this individual is most interesting since there was in the first period of iodine administration the usual and marked reduction of creatine without reduction of basal or any marked change in the clinical condition. Following the withdrawal of iodine there was a most extraordinary jump in the creatine excretion without significant change in the creatinine. When opera- 


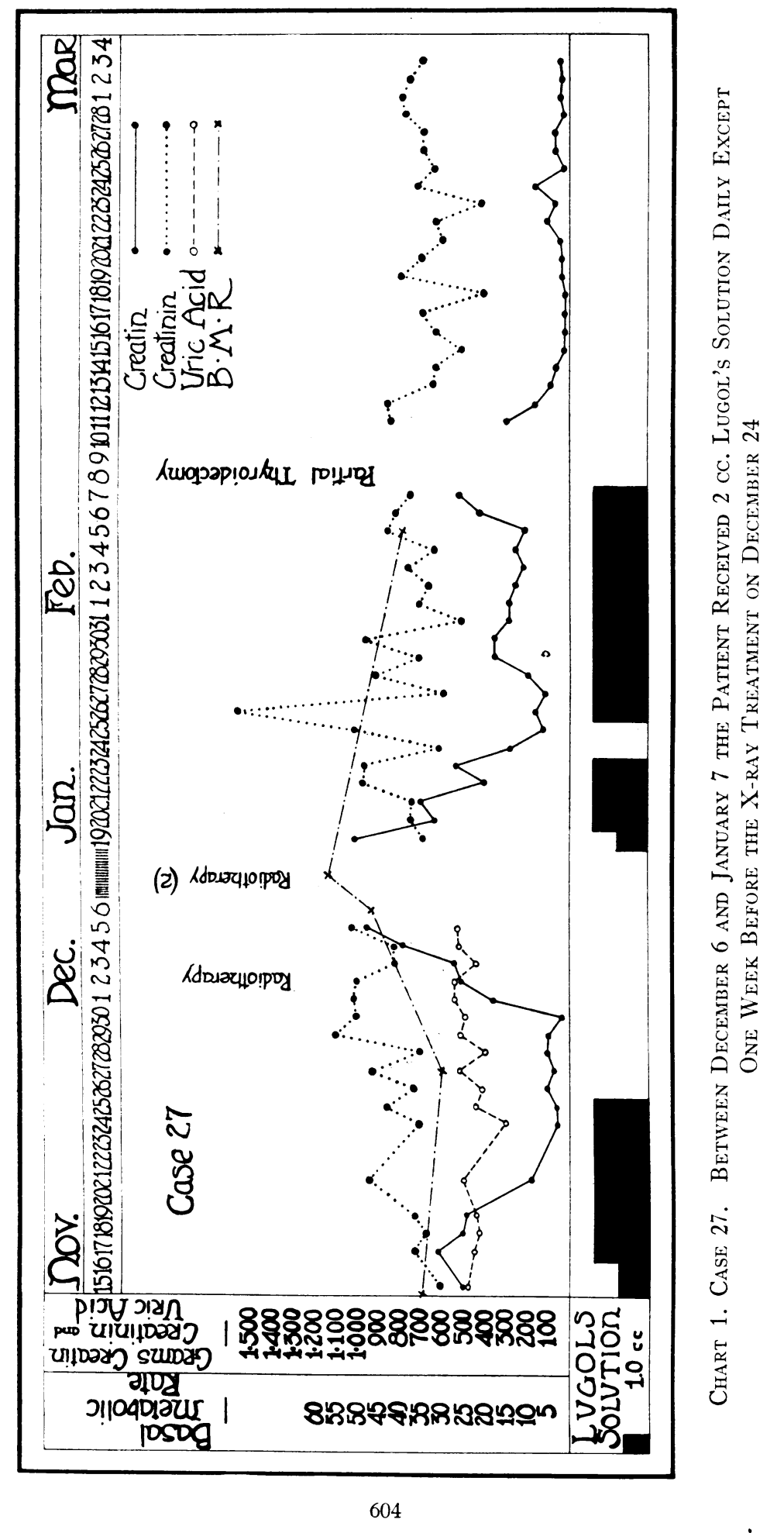


tion was refused it was decided to employ radiotherapy. A treatment was given December 2nd. The extraordinary rise in the creatine excretion might be attributed to the treatment. However, we have had an opportunity to observe the effect of radiotherapy in other cases and we are convinced that this form of treatment has little effect on creatinuria. This patient was given a second radiation on December 24th, a third on January 14th. In the interval between the periods under observation in the hospital she received $2 \mathrm{cc}$. of Lugol's solution daily excepting during the week preceding radiation. During the second period of iodine intake in preparation for operation there appears a decided reduction of the amount of creatine but with a tendency for an increase after two weeks. One is led to associate the behavior of the creatinuria in this case with the well known transitory effect of iodine in exophthalmic goiter. After operation only traces of creatine in the urine are found. No change in the creatinine excretion occurs.

Included in the data in the first iodine period is the uric acid output which is quite constant throughout. Early in the work we followed the uric acid excretion and the nitrogen balance in the subjects under observation. Iodine seemed to have no influence on the uric acid excretion. The subjects were easily brought into nitrogen equilibrium or a positive balance on a diet the caloric value of which equalled or exceeded 100 per cent above the actual basal requirement. As a result of these observations uric acid and nitrogen determinations were discontinued after the first 10 cases.

\section{DISCUSSION}

The appearance of creatine in the urine has stimulated much investigation and considerable conflicting discussion. It is not the intention of the authors to enter into the controversy at this time. The subject has been extensively reviewed by Hunter (3). Creatinuria in most instances is probably of endogenous origin due either to dissolution of muscular tissues, as in starvation, carbohydrate lack; to increased endogenous metabolism as in hyperthyroidism or to the inability on the part of the organism to utilize creatine produced normally.

Creatinuria observed in exophthalmic goiter is interesting because of the influence iodine intake has in causing creatine to disappear 
from the urine. Most of the clinical symptoms and metabolic disturbances in this disease are attributed to an increased amount of the active principle, thyroxine, circulating in the body. The evidence for such a hypothesis is considerable. Feeding thyroid gland or thyroxine to normal individuals and animals reproduces the symptoms and metabolic changes observed in the disease with the exception of exophthalmos. In our experiments we were able to bring the subjects ill with exophthalmic goiter into nitrogen equilibrium without influencing greatly the creatinuria which is interesting in connection with Benedicts and Osterberg's (5) experiments with phlorizinized dogs which were given sufficient amounts of creatine-free protein to abolish the negative nitrogen balance without diminishing the excretion of creatine. Following the administration of iodine there resulted a rapid diminution of the creatine in the urine to merely traces. In a few instances this occurred without reduction of the basal metabolism, also a few cases with high basal rates excreted very little creatine with or without iodine. As the latter phenomenon occurred most frequently in the group designated as "toxic adenomas" the question arises whether the pathological process in these cases differs in any way from the patients classified as exophthalmic goiter. There is insufficient evidence at present to answer this question. Certain it is that in those cases of hyperthyroidism with significant amounts of creatine in the urine there is marked diminution in the creatinuria following the intake of iodine. It is difficult to separate this fact from the beneficial effect of iodine in general. Marine suggests that the histological change in the hyperplastic thyroid gland brought about by iodine resulting as it does in distending the acini with colloid material, impairs the circulation in the vascular and lymphatic systems thereby preventing the escape of thyroxine into the general circulation. This view would seem to be consistent with the evidence available at present. Such being the case it would appear that thyroxine in abnormal amounts is directly associated with the appearance of creatine in the urine. In support of this conception is the fact that both in men (6) and animals (7) creatinuria is produced by feeding either the thyroid gland or the actual principle. Sturgis and associates (8) showed that the increased basal metabolism produced in rabbits by feeding thyroid extract was not lowered by the 
administration of iodine. In some unpublished experiments of the authors, rats fed thyroid extract, developed a marked creatinuria which was uninfluenced by the administration of iodine. Furthermore, we have given iodine to patients with fever and metabolic disturbances who were excreting appreciable amounts of creatine without apparent influence on the creatinuria. These facts suggest a definite relation between the disturbance in endogenous metabolism caused by hyperthyroidism and creatinuria but not necessarily one dependent on muscle dissolution.

Fiske and Subbarrow (9) have reported a labile phosphorus-creatine compound which seems to be intimately associated with normal muscle function. This observation is of great interest in connection with our studies on creatinuria in hyperthyroidism. The implication is that the phosphorus creatine compound may play an important part in normal muscular contraction since Fiske and Subbarrow found a marked reduction of the labile compound after prolonged stimulation of muscle. As one of the striking symptoms of hyperthyroidism is muscular weakness, and since one of the outstanding metabolic disturbances is creatinuria some definite relation between the two phenomena is immediately suggested. In a few preliminary experiments, however, we have not been able to establish any clear relationship. Blood phosphorus in cases of exophthalmic goiter appears to be in the upper level of normal and no significant change occurs after iodine administration. Furthermore, a single estimation of labile phosphorus in the muscle of a patient with exophthalmic goiter before iodine was given revealed about the same amount found in normal human muscle. Work on this phase of the problem is in progress and will be reported in another communication.

\section{CONCLUSIONS}

1. Creatinuria has been studied in thirty-two cases of exophthalmic goiter and eleven cases of so-called "toxic adenoma."

2. In all cases excreting appreciable amounts of creatine in the urine there is a marked diminution of the creatinuria following the administration of iodine. The influence of iodine on the creatinuria is usually accompanied by a fall in basal metabolism but not always. 
3. The relation between hyperthyroidism and endogenous nitrogen metabolism with especial reference to creatinuria has been discussed.

4. In view of the possible significance of the labile phosphoruscreatine compound in normal muscle function and the outstanding symptom of muscular weakness, the relation of the creatinuria in hyperthyroidism to these phenomena is suggested.

\section{BIBLIOGRAPHY}

1. Krause, R. A., Quart. J. Exper. Physiol., 1911, iv, 293. On the Urine of Women under Normal Conditions with Special Reference to the Presence of Creatin.

2. Denis, W., and Minot, A. S., J. Biol. Chem., 1917, xxxi, 561. The Production of Creatinuria in Normal Adults.

3. Hunter, Andrew, Physiol. Rev., 1922, ii, 586. The Physiology of Creatine and Creatinine.

4. Shaffer, Philip, Am. J. Physiol., 1908, xxiii, 1. The Excretion of Kreatinin and Kreatin in Health and Disease.

5. Benedict, S. R., and Osterberg, E., J. Biol. Chem., 1914, xviii, 195. Studies in Creatine and Creatinine Metabolism. III. The Origin of Urinary Creatine.

6. Carson, Donald A., Proc. Soc. Exper. Biol. and Med., 1928, xxv, 382. Creatine Excretion in Artificial Hyperthyroidism.

7. Unpublished experiments.

8. Sturgis, Cyrus C., Zubiran, Salvador, Wells, Guy W., and Badger, Theodore, J. Clin. Invest., 1926, ii, 289. The Effect of Iodine by Mouth on the Reaction to Intravenous Injections of Thyroxin.

9. Fiske, Cyrus H., and Subbarrow, Y., Science, 1927, lxv, 401. The Nature of the "Inorganic Phosphate" in Voluntary Muscle. 